\title{
Analytical Method Development and Validation: A Concise Review
}

\author{
Raysing Swati*1, Patil Mansi ${ }^{1}$ and Patil Aditya ${ }^{1}$ \\ ${ }^{*}$ Assistant Professor, Department of Quality Assurance, R C Patel Institute of \\ Pharmaceutical Education and Research, Karwand Naka, Shirpur, Dhule, India \\ 1Department of Quality Assurance, R C Patel Institute of Pharmaceutical Education and \\ Research, Karwand Naka, Shirpur, Dhule, India
}

Received: 10 Oct 2020 / Accepted: 8 Nov 2020/ Published online: 01 Jan 2021

*Corresponding Author Email: swatiraysing123@gmail.com

\begin{abstract}
The development and validation of analytical methods is combined with $R$ \& $D$, quality monitoring and quality assurance. Analytical techniques perform an important protagonist in risk assessment and risk management. By the analytical method development pharmaceutical industries are able to produce products of crucial characteristics and determined quality in cost effective manner. Validation is a important tool in manufacture of quality products. validation policy should complete all regulations according to good manufacturing practices (GMP). In analytical method development quality of product is determined by checking factors like accuracy, precision, Limit of Detection (LOD), limit of quantitation (LOQ), ruggedness, robustness.
\end{abstract}

\section{Keywords}

Accuracy, Linearity, Limit of quantitation, Limit of detection, Method development, Precision, Robustness, Ruggedness, Validation.

\section{INTRODUCTION}

Analytic chemistry is science division which customs innovative skills to determine work by analytical technique. ${ }^{[1]}$ It is a science which deals with physical chemistry, it is a branch of pure chemistry. ${ }^{[2]}$ Analytical system development consumes key movement of study. The topical development of analytical procedures is developed based on progress of analytical tools. Analytical procedures are innovative and validated for active drug products ingredients (APIs), pharmaceuticals, degradation products and related materials, excipients, residual solvents, etc. ${ }^{[3]}$ Drug analysis plays an important role in quality assurance as well as quality control of bulk drugs and drug formulations. ${ }^{[4]}$ Development and formulation of pharmaceutical products have led to improvements in human health. These pharmaceutical products would only be useful if they are free of impurities and if they are consumed in an appropriate quantity. ${ }^{[5]}$

Types of Analysis: There are 2 types of analysis, which are shown in fig. No. 01.

1. Qualitative analysis

Defining qualitative analysis is an approach used to better understand the underlying reasons, views and motivations. [6] Defining qualitative analysis is an approach used to better understand the underlying reasons, views and motivations. [6] Qualitative analysis may be carried out devoid of quantitative analysis, however quantitative analysis demands that analyses for which figures are given be identified (qualified). ${ }^{\text {[7] }}$

2. Quantitative Analysis

Defining quantitative analysis is an approach that quantifies the problem by generating numerical numbers or information that can be modified into usable statistics. Its purpose is to quantify categories, 
attitudes, behaviours and other variables. [6] Determining absolute or relative abundance is commonly articulated in the form of a deliberation of one, numerous or all the individual substances included in sample. When existence of a specific constituent in a sample has been identified, an investigation of its absolute or relative abundance can provision the purpose of executive belongings. ${ }^{[7]}$ The study of quality plays an especially important role in determining the quality requirements of chemical medicinal products. The number of drugs advertised in the marketplace each year. there is very often a delay between the date of introduction of a medicinal product on the market and the date of its addition to the pharmacopeia. Hence, It is possible that the criteria and methods of analysis for these medicines are not accessible in pharmacopoeias. It is therefore mandatory to breed more up-to-date analytical methods for such medicinal products. Solvents used for fabrication of medicinal constituents and excipients or active pharmaceutical ingredients (APIs) or in the formulation of drug products are frequently required. ${ }^{[8]}$

Drug analysis is important in several products and services, but in drugs it is very important because it includes life. [7] These tests may differ from a one unit or mixture of multiple powerful drugs in their formulation.

The novel pharmaceutical or mix of medications could not approved in somewhat pharmacopoeias. Analytic methods may not be available for the medicinal product as formulation excipients. Methods of analysis of a drug in grouping with additional drugs may be unavailable. A suitable trial system for drug may be unavailable in the literature because of patent regulations. There may be no analytical methods available to quantify the drug in biological fluids. Prevailing methods of analysis may necessitate high priced reagents and solvents. It could implicate a heavy removal and separation process, which may not dependable. ${ }^{[3]}$

Drug development is a very comprehensive process. including drug discovery, clinical trials, laboratory testing, animal studies and regulatory approvals. The method of analysis could be spectral, chromatographic, electrochemical, hyphen or various. ${ }^{[1]}$

\section{ANALYTICAL METHOD DEVELOPEMENT}

Development and validation of analytical methods can provide accurate, reliable, and coherent data. Procedures for developing methods are expensive, complex, and time-consuming. A method of analysis specifies the steps and techniques required for carrying out an analysis. This may include preparing samples, standards, and reagents, using equipment, using formulae to calculate the calibration curve, etc. [1] This involves developing and validating a new analytical method for resolving test samples. Samples shall be tested using UV (ultraviolet spectroscopy), IR (infrared spectroscopy), HPLC (high-performance liquid chromatography), HPTLC (High-Performance Thin-Layer Chromatography), GC-MS (Gas chromatography-Mass Spectroscopy) and LC-MS (Liquid Chromatography-Mass Spectrometer) etc. ${ }^{[3]}$

\section{The process development criteria:}

1. Trained and standardized devises

2. Recorded organization

3. Consistent indication values

4. Trained analysts

5. Experiment assortment and reliability

6. Revolution controller. ${ }^{[1]}$

The drug evaluation demonstrates the distinctiveness categorization and declaration of conjoined drugs such as dosage forms and body fluids. At a given moment in the drug generation technique and development the main resolution of analytical strategies is to generate data regarding efficacy, impurity, bioavailability, the stability and consequences of the mechanised constraints to confirm consistency in the production of the drug product prior to the development of new tools -

1. This technique has the required sensitivity?

2. Method conservative enough to work directly deprived of obstruction with the conflicting part of the sample?

3. Whether the precision of this technique is achievable?

4. Is reagents and equipment needed for this technique accessible at an affordable cost?

5. Time necessary to carry out this procedure applies? [3]

The objective of organization must duplicate development stage of the pharmaceuticals. At the beginning of drug development, methods can focus on the behaviour of APNs. It must be appropriate to provision preclinical preventive assessments, preliminary design studies and stability studies of product prototypes. So, drug development evolution, methods of analysis is advanced and prolonged to include a better understanding of API and drug products. Methodologies should be strong and straightforward, while adhering suitable official guidelines.

The method development specifies the following requirements to the analyst so that he or she can estimate the drug.

1. The required data for a given analytical problem. 
2. The required sensitivity

3. The necessary accuracy

4. The essential assortment of analysis

5. The needed precision.

Need of method development and validation.

It is recognised internationally that a developed method must necessarily be validated, since these validation methods also show the qualification and competence of the analytical laboratory.

The need for development and validation of the method of analysis since of international antagonism, preserving the standard of the product in high commercial reasons. Assorted international regulators develop standard and protocol based on the benchmark for sanction, validation and record keeping. ${ }^{[1]}$

Significance of method development and validation Significance for validation of the progress and authentication of the analytic method has appeared because of international antagonism, conserving the product customary for elevated and principled business and business motives. Numerous international regulators established the standard and protocol based on baseline for approval, authentication, and registration. ${ }^{[3]}$

Steps in Analytical Method Development - Shown in fig. no. 2.

\section{Analysis of basic categorization}

During these stage, biological and chemical characteristics (such as solubility, optical isomerism, etc.) besides the physical properties of the analytical are gathered. Afterwards this, the prevailing analysis is $100 \%$ pure is achieved and stored depending on its specific prerequisites (refrigerator, dryer, and freezer).

Once more than one component must be evaluated in the sample matrix, several numbers of components are remarked, the information is collected and accessibility of benchmark for each component is stated.

Individual methods (spectroscopic, MS, GC, HPLC, etc.) companionable by the immovability of the sample is taken into consideration. ${ }^{[3]}$

\section{Method prerequisites}

Objectives of analytic methodology that must be progressed is considered required detection limits, selectivity, linearity, range, accuracy, and precision are defined. ${ }^{[9]}$

\section{Background research and previous methods}

All information in the medication documentation is reviewed for its physical and chemical properties, manufacturing, solubility and relevant analytical methods through recommendation to significant books, journals, United States pharmacopeia/national formulary (USP/NF), association of official agricultural chemists (AOAC) and American society for examine and materials (ASTM) publications. Automated electronic document retrieval from the Chemical Abstracts Service (CAS) is convenient. ${ }^{[3]}$

\section{Choose an approach.}

The methodology is adapted in accordance with the information provided by the documentation. Procedures are modified as necessary. Additional instrumentation may be needed to procreate, moderate, enhance or validate current methodology for internal analyses and sections. Habitually a composite where the method of analysis previously subsists that is similar to the analytic of choice. ${ }^{[7]}$

\section{Instrumental configuration and analytics}

Equipment requirements are configuration Instrumentation installation, operating and implementation recommendations with laboratory SOPs are authenticated. Ever novel solvents, filters used as a test standard in appropriate injection/introduction solution and at known levels and solvents are prepared. It is imperative to edge through a familiar standard instead of convulted sample matrix. ${ }^{[7]}$

\section{Optimization}

While optimizing, single constraint is modified at a time, and all conditions are secluded, rather than resorting to a trial-and-error methodology. The job was carried out after a structured systematic strategy, and each footstep is recorded in case of impasses. [9]

\section{Documenting analytical counts of merit}

The certification of the analytic records of the services is terminated. These analytic digits of merit comprise limit of quantitation (LOQ), limit of detection (LOD), linearity, time per analysis, cost, sample for formulation. ${ }^{[3]}$

\section{Assessment of method development with real} specimens

The sample solution shall lead to an absolute and undeniable credentials of the peak of analytical interest, with exception of all former matrix modules. ${ }^{[7]}$

\section{VALIDATION:}

Validation is an integral part of analytical method development. [10] Authentication of an analytic technique shall be carried out to demonstrate its suitability for the projected purpose. Validation is carried out in demand to show that the result produced by a specific analytic experiment is reliable and inaccurate. ${ }^{[11]}$ Analytical method validation is " $A$ documented evidence, which provides a high degree of assurance that a specific process will consistently produce, a product meeting its predetermined 
specifications and quality attributes". [12] Validation is a fundamental element of quality assurance, it encompasses the efficient analysis of systems, accommodations and procedures directed at regulating whether they perform their anticipated purposes adequately and unfailingly as stated. [13] Types of validation shown in fig. no. 3 .

Kinds of analytic techniques that require validation. The examination on validating analytical techniques covers the four most communal varieties of analytical methods.

1. Identification experiments

2. Quantitative testing of impurity levels

3. Restrict testing for contamination control.

4. Quantifiable testing of a dynamic fraction in samples of a drug substance. ${ }^{[14]}$

Validation is classified in following types: ${ }^{[3]}$

Methods for analysis must be validated or revalidated.

I. Prior to introducing them into regular use

II. Each time situation alters for which the procedure has been verified. (e.g., a system with differ in qualities or samples with different matrices.)

III. Each time the method is modified, and the modification is outside the initial possibility of the method. ${ }^{[3]}$

Analytical procedure and validation - ICH Guidelines (ICH Q2R1)

The analysis process is related to how to perform the analysis. It must designate in point the steps crucial to accomplish each analytical experiment. This could incorporate but is not regulated to:

1. Sample

2. The benchmark and the reactant preparations

3. Use of the apparatus

4. producing the calibration waveform

3. Use of formulas for calculation, etc. [11]

\section{Objective of validation}

From a written procedural beginning for the production and control of processes that are designed to ensure that drug products have identity, quality, and purity.

Two primary objectives of validation: -

1) Assurance of quantity

2) Government regulation.

Validation of Method Developed

METHOD DEVELOPEMENT PARAMETERS

System suitability is expressed as, monitoring of a system, already or through analysis of nonentities, to certify system implementation. ${ }^{[15]}$

Method development parameters are shown in fig. no.4.

\section{Precision:}

The accuracy of an analytic experiment conveys the proximity of an contract between a sequence of dimensions achieved from two or more samples of the same homogeneous sample under the recommended situations. It may be further segmented into repeatability, intermediate accuracy and repeatability. The standard deviation, the relative standard deviation such as the coefficient of variation and the confidence interval must be indicated for individually type of precision examined. [16] The accuracy of an analysis method is commonly stated as variance, standard deviation or coefficient of variation of a measurement sequence.

a) Repeatability

The precision is expressed in the constant working environments over a short time interval. It is also designated as intra assay precision.

b) Intermediate Precision

It articulates indoors - deviations of laboratories like different days, different analysts, different equipment, etc.

c) Reproducibility

It expresses inter-laboratory precision (collaborative studies, typically applied to standardization of methodology). ${ }^{[3]}$

\section{Accuracy:}

Affection of settlement among the standards observed. The accepted value as a conventional true rate or the accepted benchmark assessment. Numerous approaches can be used to determine accuracy. It might be tested by the employment of an analytic experiment by an analyte of known concentration, by assessment of the outcomes of the propositioned analytic experiment with those of a second acknowledged technique, the accuracy of which is mentioned and expressed. It may also be deduced once the precision, linearity and specificity have been founded. ${ }^{[1]}$

\section{Limit of Detection:}

Limit of detection for a single analytical process is the smallest amount of analyte in a sample that can be discovered, but not automatically computed as an precise value. ${ }^{[17]}$

\section{Limit of Quantitation:}

The limit of quantification of a specific analytical technique is smallest amount of analyte in a sample that can be resolute quantitatively with appropriate precision. ${ }^{[3]}$

\section{Specificity:}

The capacity to unambiguously evaluate analytics in the existence of constituents that could be present. Typically, it can comprise of impurities, breakdowns, matrix. Deficiency of specificity of an particular 
analytic experiment might be rewarded by supplementary analytical method(s). [12]

6. Linearity:

Linearity of a method is measure of how well a calibration plot of response vs. concentration approximates a straight line. The calibration curve was plotted against concentration vs. peak area by the prepared different aliquots. ${ }^{[4]}$ Its ability (in determined range) to achieve assay results that are straightforwardly comparative to the deliberation (quantity) of the analytical sample. ${ }^{[11]}$

\section{Range:}

The range of analytical method is the interval between the upper and lower levels of analytic that has been demonstrated to be determined with a suitable level of accuracy, precision, and linearity. The range is normally expressed in the same units as test results (e.g., percent, parts per million) obtained by the analytical method. [21]

Ranges: -

I. For Assay - 80 to $120 \%$ of test focus

II. Substance consistency - 70 to $130 \%$ of test focus

III. Disintegration - Q-20 \% to $120 \%$

IV. Polluting influences - announcing level - $120 \%$ of debasement determination limit.

V. Measure and contaminations - Reporting level to $120 \%$ of examine explicit.

VI. Linearity constrained to $150 \%$ of time span of usability detail of debasements.

VII. Test fixation can be utilized to decide contaminations to decide tranquilize substance (examine) the test fixation must be weakened.

VIII. The range is $0-\sim 150 \%$ of debasement detail. [20]

\section{Robustness:}

Measurement of its ability not to be affected by minor but measured changes in procedure limits and specifies an suggestion of its consistency throughout usual practice.

Standards of characteristic adaptions - Extraction time, Stability of analytical solutions.

In liquid chromatography -

Patterns of distinctive deviations are:

I. Effect of $\mathrm{pH}$ changes during a mobile phase.

II. Effect of changes in the composition of the mobile phase.

III. Altered columns.

IV. Temperature

V. Flow rate

In the case of gas-chromatography, examples of typical variations are:
I. Temperature

II. flow rate
III. Different columns (different lots and/or suppliers). ${ }^{[18]}$

\section{Ruggedness:}

The ruggedness of an analytical method is the degree or measure of reproducibility under altered situations such as different reagents, different analysts, instruments, laboratories, temperature, time. ${ }^{[19]}$

\section{Advantages of analytical method validation:}

1. The largest advantage of method validation is that it forms a degree of confidence, not only for the developer but also to the user. ${ }^{[22]}$

2. Minimum batch product failure, enhanced efficiency, manufacturing, and productivity. ${ }^{[19]}$

3. Minute alterations in environments such as reagent provider or grade, the analytical installation is compulsory for intentions of appeasement, but the validation of the method absorbs the tremor of such situations and compensations more than authorized on the procedure. ${ }^{[22]}$

4. Each analysis technique has its own characteristics, it is also important, which can differ from one analysis to another. ${ }^{[3]}$

\section{STABILITY}

It is also an important aspect of method development. The validation stability of the method is studied under different conditions. In stability experiments, the conditions used must reflect situations which may be tested during the handling and analysis of the samples. ${ }^{[23]}$

\section{CONCLUSIONS}

The Developing analytical procedures for the resolution of chemical classes was one of the most dynamic features of the 1990s in analytical chemistry. The development of analytical procedures assists to empathise the precarious constraints of the process and to diminish their impact on accuracy and precision. It composites a key role in innovation, development, and production of pharmaceutical products. The authorized assessment procedure that outcomes from these activities are expended by quality control test centers to confirm the identity, purity, potency, and performance of drug product 'quality', necessary for drug safety and efficacy.

The test method needs to be validated. The pharmaceutical industry continues to press to reduce cycle times and increase the number of products entering the market. Once the methods have been developed, qualified and validated, their influence on non-specification rates and the capability of the process must be quantified and estimated to determine its efficiency for prospect procedure. 


\section{ACKNOWLEDGEMENT}

Authors of this article are thankful to Dr. S. J. Surana, Principal, Dr. S.S.Chalikwar HOD and Management of
R. C. Patel Institute of Pharmaceutical Education and Research, Shirpur for providing the library facility for compilation of this review article.

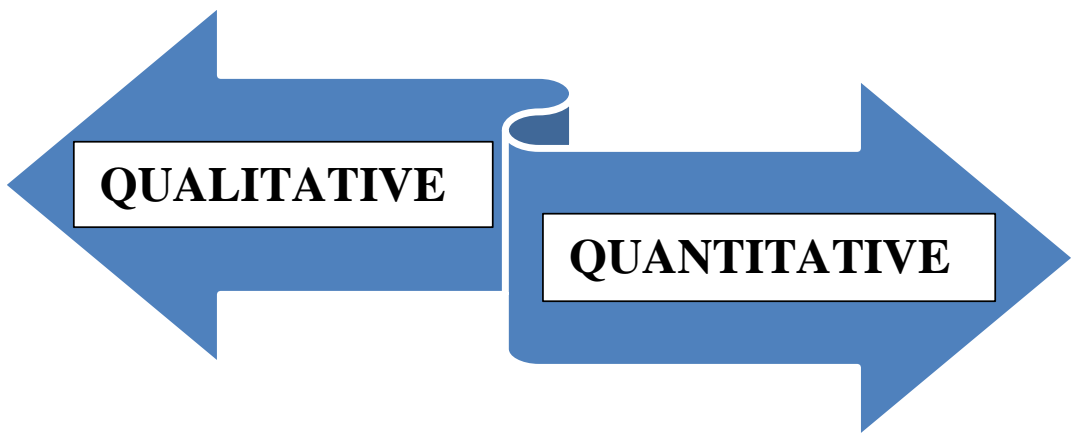

Fig.1 Types of analysis

Analyze basic categorization.

Method prerequisites

Background research and previous method

Choose an approach.

Instrumental configuration and analyte

Optimization

\section{Documenting analytical counts of merit}

Assessment of method development with real specimens

Fig 2. Steps in method development 


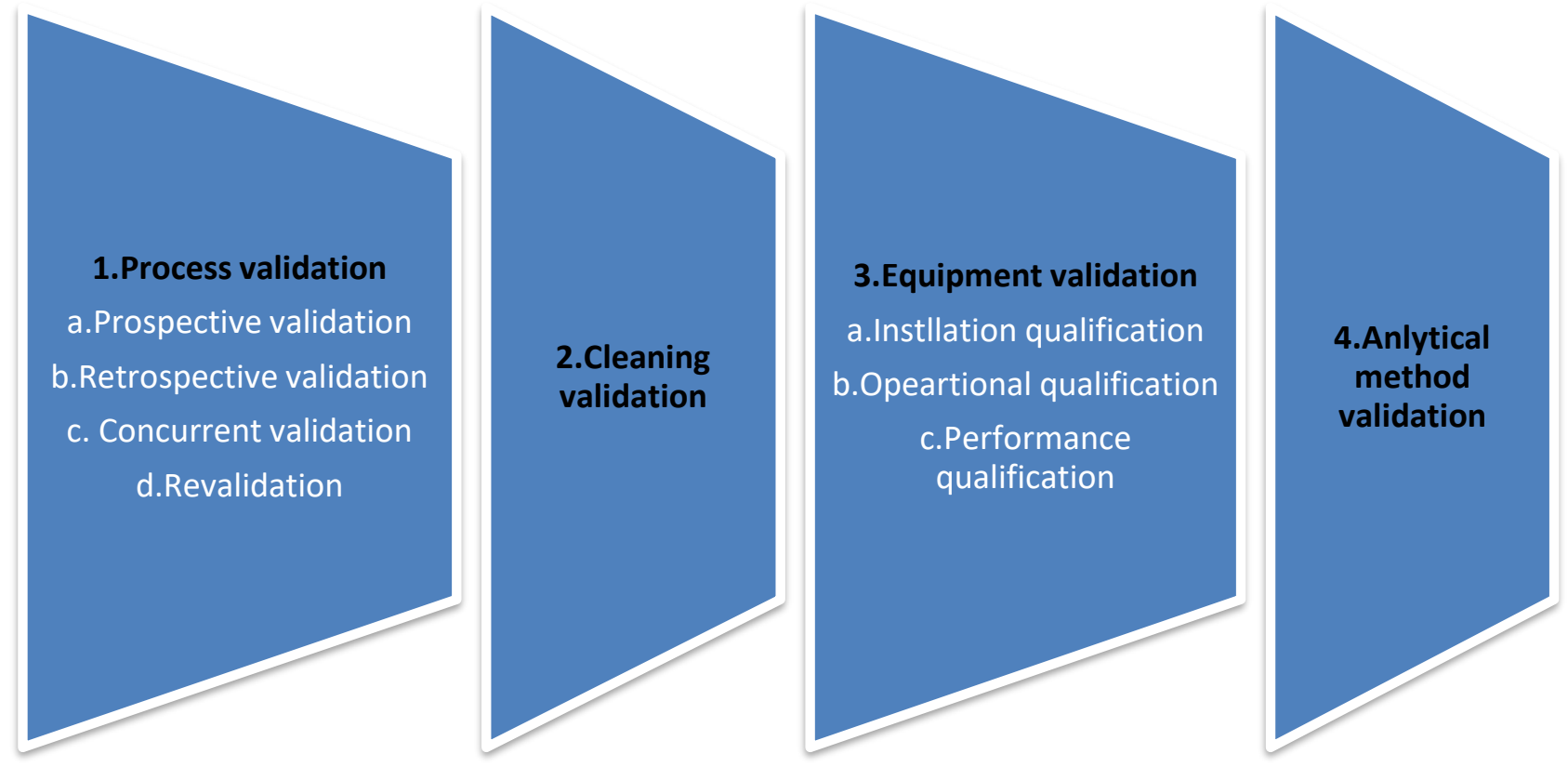

Fig 3. Types of validation

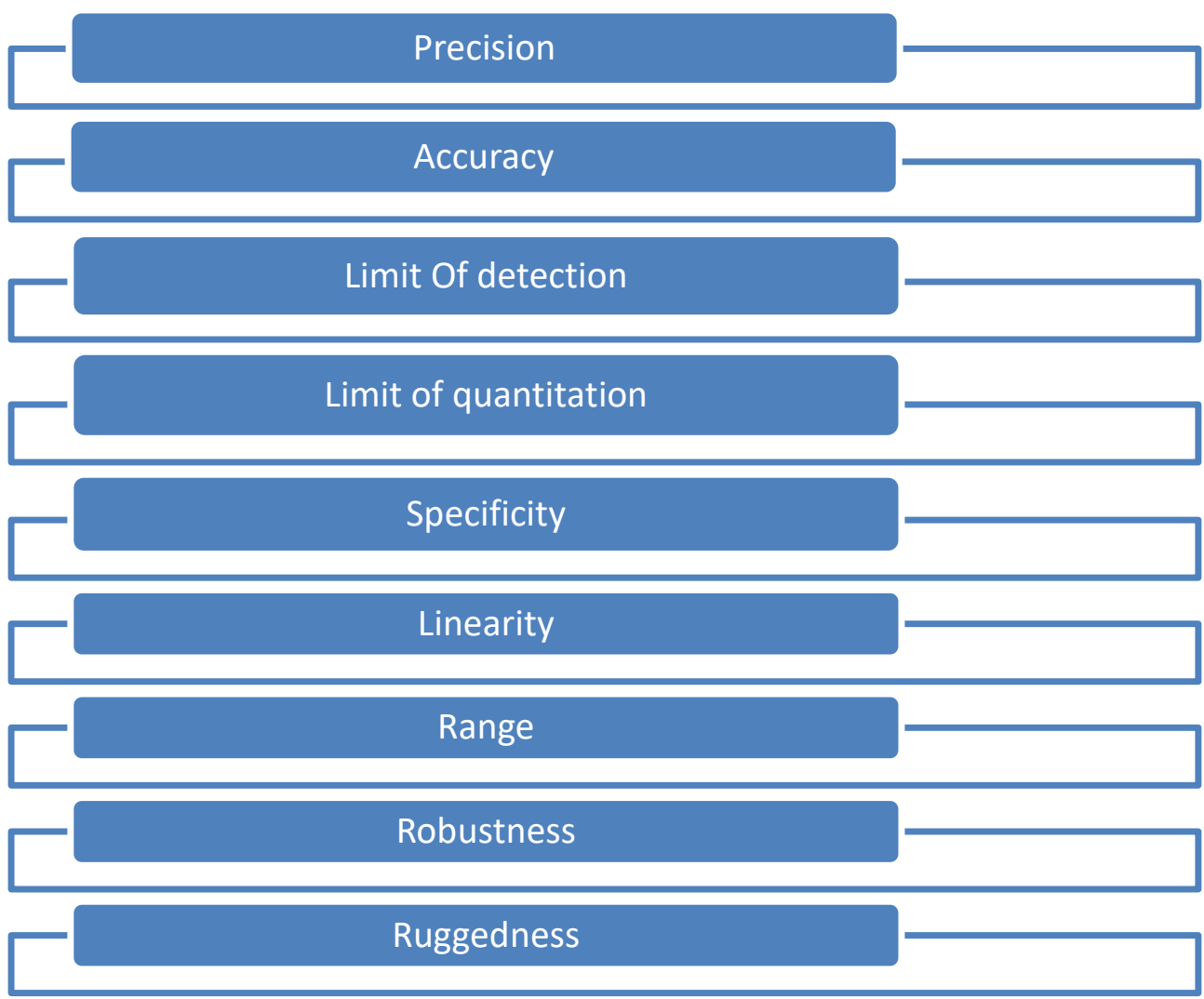

Fig. 4 Validation parameters

\section{REFERENCES}

1. Chauhan A., Mittu B.,Chauhan P.,: Analytical Method Development and Validation: A Concise Review: J Anal Bioanal Tech 6(1):1-3(2015).
2. Pasbola k., Chaudhary M.,: Updated review on analytical method development and validation by HPLC: 6(5):1612(2017).

3. Doltade M., and Saudagar R.,: Analytical method development and validation: A review: 9(3): 563(2019). 
4. P. Ravi Sankar, V. Swathi, P. Srinivasa Babu: Development and validation of novel UV and RP-HPLC methods for determination of cilnidipine (A new generation Ca channel blocker) In pharmaceutical dosage form: 10(4):1889(2019).

5. Veena D. Singh, S.J. Daharwal, Preeti K. Suresh. A review of instrumental analytical methods to assay active ingredients in multicomponent pharmaceutical formulations: (2014).

6. Sinaga A., Difference between qualitative and quantitative analysis and how it should be applied in our research: (2014).

7. Hema, Reddy S.: A review on new analytical method development and validation by RP-HPLC: 4(3):41,42,47, (2017).

8. Ameen F., Dr.Balaram M., Dr.Koneru A.,: Method developement and validation for estimation of class II solvents in dasatinib by HSGC-FID: 6(11):302(2019).

9. Ravisankar P., Gowthami S., Rao D., A review on analytical method development: 2(3):1184(2014).

10. Kumar J., Rohman H., Babu N., Babu S., Reddy R., Development, and validation of RP-HPLC method for quantitative panobinostat in pure and pharmaceutical dosage form: 8(3):991(2019).

11. Behera S., Ghanty S., Ahmad F., Santra S., and Banerjee S., UV-Visible spectrophotometric method development and validation of assay of paracetamol tablet formulation: 3(12):3948(2012).

12. Thete P., Saudagar R.,: Review on analytical method developement and validation by RP-HPLC: (5)5:4898,4899,4901,4802,4903(2018).
13. Jatto E., Augustine O., Okhamafe,.: An overview of pharmaceutical validation and process controls in drug development: 1(2):116(2002).

14. Kumar A., Kishore L., Kaur N., Nair A., Method development and validation: Skills and tricks: 3(1):5(2012).

15. Vinutha Kommineni, K.P.R.Chowdary, S.V.U.M. Prasad,: development of a new stability indicating RPHPLC method for simultanious estimation of metformin hydrochloride and teneligliptin hydrobromide and its validation as per ICH guidelines:4(5):1112(2017).

16. Suresh Kumar S., Latif D.Jamadar, Krishnamurthy Bhat, Prashant B., Musmade, S. G., Vasantharaju, N. Udupa.,: Analytical method developement and validation for aspirin: 2(1):391(2010).

17. Parekh H., Chokshi P., Mashru R., Analytical method development and validation for the estimation of sugammadex: 10(1):53(2020).

18. ICH, Q 2 (R1). Validation of analytical procedures: Text and methodology: 2005

19. Sharma S., Goyal S., Chauhan K., A review on analytical method development and validation: 2(1):12,13(2010).

20. Hashem H., Review on Analytical Method Development and Validation: 1(2):(2020)

21. Eldin A., Validation of an analytical procedure: 18(2011)

22. G. Lavanya, M. Sunil, M.M. Eswarudu, M. Chinna Eswaraiah, K. Harisudha, B. Naga Spandana.,: Analytical method validated, An updated review:4(4):1282,1285(2013).

23. Tijare LK, Rangari NT, Mahajan UN: A Review on bioanalytical method development and validation: 9(3):8(2016) 